\title{
Retrospective evaluation of childhood paraphenylenediamine intoxication due to black henna
}

\author{
Elif Güdeloğlu ${ }^{1,2}$, Barış Erdur $^{1 \oplus}$ \\ ${ }^{1}$ Department of Pediatrics, Dr. Behçet Uz Children's Hospital, İzmir, Turkey; ${ }^{2}$ Nyala-Sudan Turkey Training and Research Hospital, \\ Nyala, Sudan.
}

\begin{abstract}
Background and objectives. Paraphenylenediamine (PPD) is a toxic substance in henna. Oral intake of this substance causes severe systemic toxicity. To the best of our knowledge there are no studies in the literature conducted only on children exposed to henna intoxication.
\end{abstract}

Methods. Twenty-three patients aged between 1 and 17 who referred to Nyala-Sudan Turkey Training and Research Hospital between May 2015 and June 2018 were evaluated retrospectively in terms of demographic, clinic and laboratory characteristics.

Results. Four (17.39\%) patients were male and 19 (82.61\%) were female. Average age of patients was $10.95 \pm 3.2$. Most of the referrals to the hospital following PPD intoxication occurred in the first 16 hours. All of the patients between 10 and 17 had taken henna for suicidal purposes. All patients had vomiting and agitation. The most common symptoms apart from these were gastrointestinal symptoms, tachycardia, tachypnea and dyspnea. Twelve (52.17\%) patients had elevated liver function tests and $3(13.04 \%)$ had developed renal failure. None of the patients had neurological complications. Two (8.70\%) patients developed a need for tracheostomy. Average hospitalization period of patients was 8.5 days. Two patients died. One was in 1-5 age group and died due to renal complications, while the other was in 6-10 age group and died due to hepatic failure.

Conclusion. PPD intoxication is a life-threatening situation even in low doses. For this reason, even asymptomatic cases should undergo physical examination and should be followed closely in terms of respiratory tract obstruction. Ensuring hydration and diuresis in the early period, steroid and adrenalin therapy for prophylaxis in terms of respiratory tract obstruction are important and tracheostomy should not be abstained in necessary cases. It should not be forgotten that symptomatic treatment for organ systems and dialysis will decrease mortality and morbidity.

Key words: childhood, paraphenylenediamine, henna, intoxication.

Paraphenylenediamine (PPD) is a toxic substance found in henna which is used as traditional dye to give hair a dark color. Accidental or suicidal intake of henna causes severe systemic toxicity. ${ }^{1}$ The primary substance giving rise to this situation is PPD. ${ }^{2}$ Toxicity caused by henna is very common in Sudan. Globally, suicidal deaths have been found to increase $60 \%$ in the last 50 years. ${ }^{2}$ Large-scaled studies have shown that PPD is responsible for

$\triangle \quad$ Elif Güdeloğlu

drelif55@hotmail.com

Received 22nd November 2018, revised 4th November 2019, accepted 24th November 2019.
$35 \%$ of suicidal deaths in Sudan. ${ }^{3}$ The high rate of suicide with henna can be due to the fact that it is cheap and easily accessible or owing to previous suicide experiences.

Clinical course is various in toxications with PPD. However, there is no antidote and the intoxication may lead to mortality unless early intervention is initiated. ${ }^{4}$ Although the lethal dose has been reported as 10 gram after oral intake and enteral absorption of PPD, it has also been reported lethal even in very small doses in some cases. ${ }^{3}$ Suicidal henna intoxication generally occurs due to oral intake. The main part of the treatment consists of supportive treatment and it is an emergent medical 
condition. Symptomatic treatments include providing airway patency, endotracheal intubation, and tracheostomy if necessary, gastric lavage, providing fluid electrolyte balance and hemodialysis in case of renal failure. ${ }^{2,4,5}$ To the best of our knowleadge there are no studies in literature evaluating exposure to henna intoxication including the pediatric age group only. The aim of this study was to evaluate the effects of henna intoxication in children.

\section{Material and Methods}

The present study protocol was approved by Nyala Sudan- Turkey Education and Research Hospital Ethics Commitee's (NSTH.03/903.07.03-09).

This study was a retrospective cohort study and files of 23 patients aged between 1 and 17 years who were referred to the NyalaSudan Turkey Training and Research Hospital between May 2015 and June 2018 were reviewed retrospectively. The patients were divided to three groups according to age as group 1; 1-5 years, group 2; 6-10, and group 3; 11-17 years. Patients' gender, marital status, pregnancy, reason for intoxication (suicidal/ accidental/homicidal) were questioned. The time of referral to hospital was grouped as 0-8 hours, 9-16 hours, and 17 hours and more. Laboratory parameters; complete blood count, serum biochemistry (urea, creatinine, aspartate transaminase (AST), alanine transaminase (ALT), total bilirubin, direct bilirubin), serum electrolytes (sodium, potassium, chlorine, calcium), coagulation parameters; prothrombin time (PT), international normalized ratio (INR), activated partial thromboplastin time (aPTT), , blood gas $(\mathrm{pH}$, partial carbondioxide pressure, bicarbonate, anion gap) and total urine test were evaluated based on laboratory reference values of the hospital. Electrocardiogram (ECG) records were obtained from all patients. The patients were questioned for psychiatric diagnoses, known before referral for intoxication and evaluated in terms of mortality and morbidity. Complications were grouped as cardiovascular, respiratory, gastrointestinal, neurological, and renal. The methods of treatment for complications were grouped as medical treatment (adrenalin, steroid, hydration and diuresis), surgical treatment, and dialysis. All statistical analysis was conducted with the SPSS (Statistical Package for Social Sciences) for Windows version 19.0 release (IBM, Chicago, IL). Frequency and averages were calculated by using descriptive statistic tests.

\section{Results}

Of the 23 patients, 4 (17.39\%) were male, 19 $(82.61 \%)$ were female. There were $2(8.70 \%)$ patients in 1-5 years age group 1, $8(34.78 \%)$ in 6-10 years age group 2, and 13 (56.52\%) in 11-17 years age group 3 . Average age was $10.95 \pm 3.20$ years. Three $(23.07 \%)$ patients in group 3 were married, while 10 (76.92\%) were single. One patient was pregnant. Average hospitalization period was $8.48 \pm 1.12$ days. In 13 (56.52\%) of the patients' hospitalization time was more than 9 days. Demographic, epidemiological and clinical data of the patients are given in Table I. The patients mostly referred to the hospital within 16 hours following PPD intoxication (Table I). Only 4 had referred to the hospital in 17 hours and more. Average period of referral was $8.78 \pm 1.9$ hours. All the patients in group 3 had ingested henna for suicidal purposes, while the others had ingested accidentally. The way of henna ingested was oral in all patients. None of the patients had previous psychiatric diagnosis. It was found that symptoms of henna toxication started 3 hours or longer in $47.83 \%$ of the patients after ingestion, and $76.92 \%$ of these patients were in group 3 . The symptoms started with in the first hour of intoxication in all the patients of the group 1, and $75 \%$ of the group 2. Vomiting and agitation were observed in all patients. The other most common symptoms were gastrointestinal symptoms, tachycardia, tachypnea and dyspnea. All children in group 1 had tachycardia, tachypnea and dyspnea. Angioedema was not observed in group 1 but it was found in $7(30.43 \%)$ patients in other 
Table I. Demographic, epidemiological and clinical data of the patients.

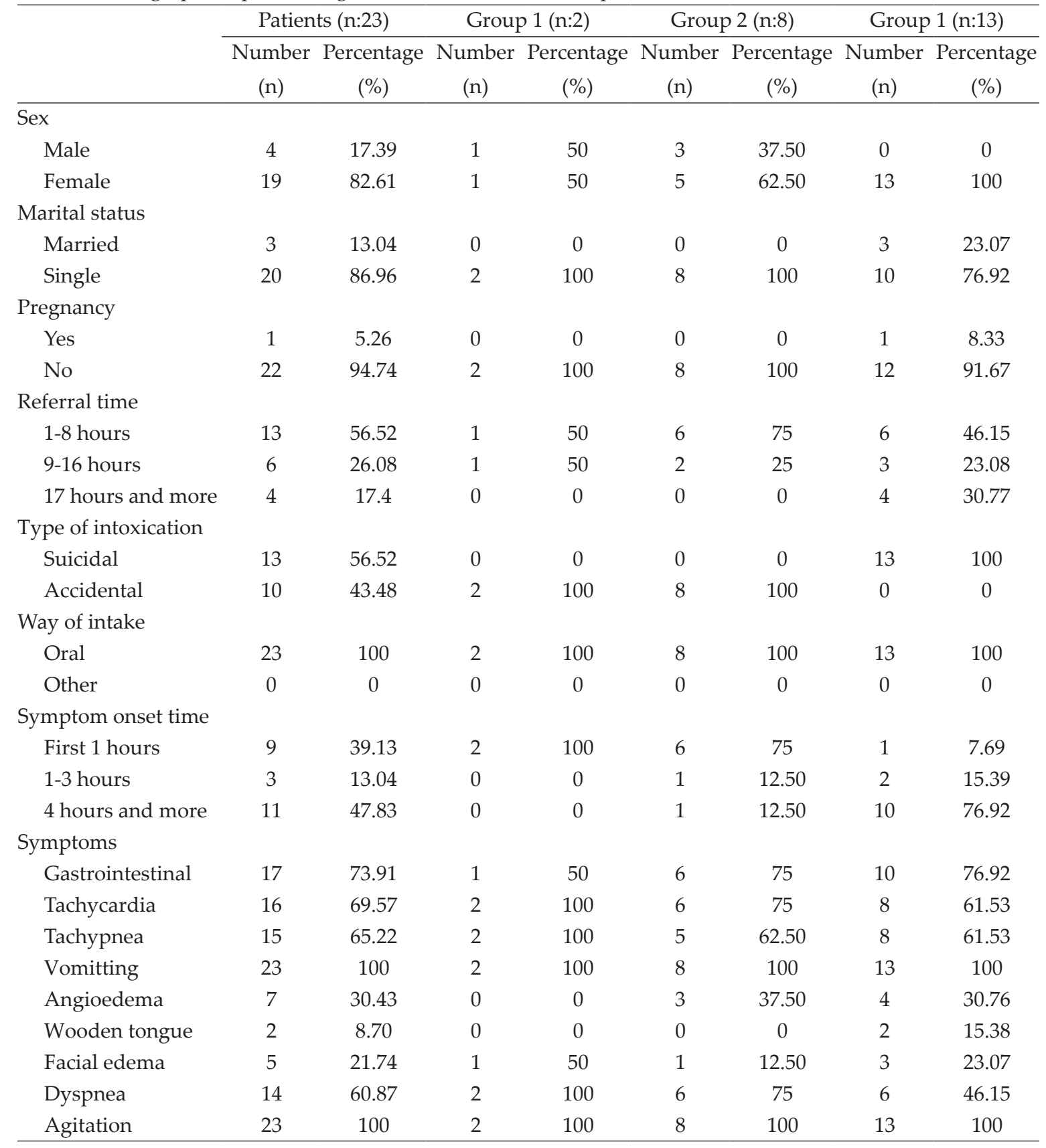

${ }^{*} \mathrm{n}$ : Number of patients

age groups. Wooden tongue was found only in 2 patients in group 3. Laboratory data of the patients was given in Table II. Twelve $(52.17 \%)$ patients had elevated liver function tests, and in 3 of them (13.04\%), the values were twice that of the normal level. The blood urea and creatinine levels were high in 7 (30.43\%) patients. Metabolic acidosis nonresponsive to treatment and renal failure requiring dialysis developed in $3(13.04 \%)$ of the 7 patients. Serum electrolyte imbalance (imbalance in at least one of the serum sodium, potassium, chlorine, calcium values) was observed in $5(21.73 \%)$ patients. In terms of complete blood count 
Table II. Laboratory test results of the patients.

\begin{tabular}{lcc}
\hline & \multicolumn{2}{c}{ Patients (n:23) } \\
\cline { 2 - 3 } Laboratory tests & $\begin{array}{c}\text { Number } \\
\text { (n) }\end{array}$ & $\begin{array}{c}\text { Percentage } \\
(\%)\end{array}$ \\
\hline $\begin{array}{l}\text { Serum biochemistry } \\
\quad \text { Elevated transaminases }\end{array}$ & 12 & 52.17 \\
$\quad$ Elevated urea/creatinine & 7 & 30.43 \\
$\quad$ Electrolyte imbalance & 5 & 21.73 \\
Coagulation & & \\
$\quad$ Elevated INR** & 9 & 39.13 \\
Complete blood counting & & \\
$\quad$ Leucocytosis & 6 & 26.08 \\
$\quad$ Eosinophilia & 8 & 34.78 \\
$\quad$ Thrombocytopenia & 3 & 13.04 \\
Urine analysis & & \\
$\quad \begin{array}{l}\text { Proteinuria, hematuria, } \\
\text { glucosuria }\end{array}$ & 6 & 26.08 \\
\hline
\end{tabular}

*n: Number of patients

**INR: International normalized ratio

parameters, leukocytosis was observed in 6 (26.08\%) patients, eosinophilia was found in 8 $(34.78 \%)$ patients, and thrombocytopenia was observed in $3(13.04 \%)$ patients. Nine (39.13\%) patients had elevated international normalized ratio (INR) values. Proteinuria, glucosuria and hematuria were observed in urine tests of $6(26.08 \%)$ patients. ECG of $3(13.04 \%)$ patients showed prolonged PR interval. Table III demonstrates treatment methods, complications and mortality of the patients. Seven $(30.43 \%)$ patients had renal, 7 (30.43\%) patients had respiratory, $3(13.04 \%)$ patients had cardiovascular, and $3(13.04 \%)$ patients had hepatic complications. None of the patients had neurological complications. All patients received a nasogastric catheter and gastric lavage was performed. Methylprednisolone was given to all patients, as was intravenous (IV) hydration and furosemide treatment to increase diuresis. Four $(17.39 \%)$ patients received intramuscular (IM) adrenalin, 10 (43.47\%) patients received symptomatic treatment for cardiopulmonary symptoms. Tracheostomy was performed on 2 $(8.70 \%)$ patients. All patients were followed in the intensive care unit on the first three days. One patient (4.34\%) in group 1 had died due to
Table III. Data of treatment options, complications and mortality of the patients.

\begin{tabular}{|c|c|c|}
\hline \multirow{3}{*}{ Treatment } & \multicolumn{2}{|c|}{ Patients (n:23) } \\
\hline & Number & Percentage \\
\hline & (n) & $(\%)$ \\
\hline \multicolumn{3}{|l|}{ Medical } \\
\hline Gastric lavage & 23 & 100 \\
\hline Methylprednisolone & 23 & 100 \\
\hline Hydration & 23 & 100 \\
\hline Furosemide & 23 & 100 \\
\hline Adrenalin & 4 & 17.39 \\
\hline $\begin{array}{l}\text { Symptomatic treatment for } \\
\text { cardiovasculary symptoms }\end{array}$ & 10 & 43.47 \\
\hline Surgery & - & - \\
\hline Tracheostomy & 2 & 8.70 \\
\hline Dialysis & 3 & 13.04 \\
\hline \multicolumn{3}{|l|}{ Complications } \\
\hline Renal & 7 & 30.43 \\
\hline Respiratory & 7 & 30.43 \\
\hline Cardiovasculary & 3 & 13.04 \\
\hline Hepatic & 3 & 13.04 \\
\hline Neurologic & 0 & 0 \\
\hline \multicolumn{3}{|l|}{ Mortality } \\
\hline Renal failure & 1 & 4.35 \\
\hline Hepatic failure & 1 & 4.35 \\
\hline Total & 2 & 8.70 \\
\hline
\end{tabular}

the renal complications, and one patient (4.34\%) in group 2 died due to the hepatic failure.

\section{Discussion}

Paraphenylenediamine (PPD) is the main toxic substance in henna which is used for dying hair, hands and nails in Sudan. ${ }^{1,2}$ PPD is an oxidative chemical; intoxications with this substance can cause hypersensitivity reactions and systemic side effects. ${ }^{6-8}$ While there are studies about toxications with several substances in different geographies in Africa, suicidal PPD intoxications have been reported in Eastern African countries such as Sudan. ${ }^{8}$

Similar to the previous literature, most cases, especially in older children were suicidal. In Sudan, high rate of suicide was associated 
with difficulty of life conditions, stress, low socioeconomic level, strict cultural rules, and psychological problems. ${ }^{3}$ It has been reported that single people were frequently exposed to PPD intoxication, and attempt for suicide when compared with the married ones. ${ }^{3}$ One of our patients was exposed to PPD intoxication in pregnancy. With early and effective treatment, neither the baby nor the mother had any complications. There is insufficient data about PPD intoxication in pregnancy in the literature.

The average hospital referral time was 8.78 \pm 1.9 hours. In PPD intoxication, symptoms generally occur within the first hours, and may result in mortality and morbidity. Hospital referral times of the patients were relatively late in our study. Late referral time was considered to be associated with low socio-economic and education level, and transportation difficulties to emergency departments of hospitals.

In some patients, tracheostomy may be required due to obstruction in upper airway. The need for tracheostomy has been reported in higher rates for both adults and children in literature. ${ }^{9-11}$ The patients who applied to our hospital after 17 hours or more due to intake of PPD was for $17 \%$ (4 patients). Two of the four patients applied to the hospital between 17 and 24 hours, and they did not need tracheostomy. The other two patients were admitted to our hospital after 48 hours, with severe respiratory obstruction findings and they underwent tracheostomy. In our study, the low number of patients with tracheostomy was considered to be due to a few number of patients admitted after 24 hours. This situation gives rise to thought that early admission and effective treatment may be related to decreased tracheostomy need.

In a study of 200 patients from Sudan, it was found that PPD intoxication peaked between 14 and 27 years of age and was generally taken for suicidal purposes. ${ }^{3}$ In our study, PPD toxicity was observed more frequent in group 3. Similar to the other studies, in our study PPD was mostly taken orally. Other studies have shown that after high amount of henna intake for suicidal purposes, symptoms started generally within one hour. ${ }^{3}$ Whereas symptoms started later (three or more hours) in our study. In Africa, especially in undeveloped countries such as Sudan, difficult economic conditions and low education levels have unfavorable effects to life styles of people. For this reason, many people try to overcome from their many medical conditions by themselves without hospital admission. In association with their religious beliefs, which is extremely fatalistic, African people have high tolerans levels when compared with western people, who usually prefer refer to seek medical care immediately. Older patients try to tolerate their complaints such as pain due to their beliefs as a result of their culture, and express these only when they become unbearable. In the younger age group (group 1 and 2), symptoms started in the first hour, similar to literature. Although there are no studies in the literature covering patients only in pediatric age group, an adult study including pediatric patients demostrated that accidental intake was more frequent in the pediatric age group. ${ }^{9}$ The behaviour of recognizing objects by taking them into mouth especially within the first 3 years of age plays the main role in accidental PPD intake. There were also studies reporting that suicide rate was higher in puberty. ${ }^{12,13}$ While the rate of suicidal PPD intake was $56.52 \%$ patients in our study, the rate of accidental intake was $43.48 \%$.

Most frequent problems following PPD intoxication were reported as upper respiratory tract problems, hypersensitivity reactions, rhabdomyolysis resulting in renal failure, cardiac disorders presenting with dysrhythmia and liver disease presenting with elevated liver transaminases. Similar to the other studies, in our study, respiratory diseases were the most common complication which was followed by renal, cardiovascular, and lung complications. ${ }^{3,8,9}$ In $82 \%$ of patients exposed to PPD, chocolate brown dark urine was reported due to hemolysis and rhabdomyolysis. While there are studies in literature reporting dialysis need following oliguria in adult patients 
exposed to PPD, $3(13.04 \%)$ of the patients received dialysis in our study. ${ }^{9}$ In De Groot's study ${ }^{14}$, it was reported that allergic contact dermatitis with the use of hair dyes including PPD or related chemicals could develop and such reactions could require hospitalization especially in children. Since cross-reactivity can develop for other hair dyes, textile paints, local anesthetics and rubber chemicals in most of those sensitized to PPD, contact with these materials should also be avoided. ${ }^{14}$ It was reported that due to increased use of temporary black henna tattoos and inadequacy in the legal control of henna tattooing practice, an increase in PPD sensitization cases would be inevitable in the future and thus black henna tattooing should be controlled by health authorities. ${ }^{14}$ However, it was remarkable in our study that PPD toxicity induced skin symptoms following oral intake were not seen in any of our patients.

The main steps of the treatment following PPD intoxication are providing continuation of airway, breathing, and circulation, gastric lavage following by nasogastric tube placement, hydration and providing forced diuresis to decrease the intensity of toxic substance in the plasma, close monitorization and symptomatic treatment in the intensive care unit for possible complications. ${ }^{9}$ Ten $(43.47 \%)$ patients received treatment for cardiovascular symptoms, $4 \quad(17.39 \%)$ patients received adrenalin for severe angioedema not responding to methylprednisolone, $2(8.7 \%)$ patients were applied tracheostomy for establishment of a more stable airway, and $3(13.04 \%)$ patients received dialysis. Antihistamines and steroids are commonly used in the management of airway edema because of the possibility of a hypersensitivity reaction to PPD but there is no clinical evidence to support this type of treatment. ${ }^{15}$ Also, in our study severe angioedema did not respond to steroids and we had to use adrenaline for treatment. In case of repiratory system complications, tracheostomy is a life saving procedure. ${ }^{16}$
In 4 studies including all age groups mortality rates were found as $33.30 \%, 11.90 \%, 9.50 \%$, and $3.50 \%{ }^{3,17-19}$ These lower rates were explained with early referral to hospital and detoxification treatment given to patients. ${ }^{3,17-19}$ In a study by Elgamel et al. ${ }^{3}$, mortality occured due to respiratory obstruction in $71.40 \%$ of the patients, and renal failure in $28.60 \%$ of the patients additionally mortality rate was found higher in the pediatric age group. In our study, mortality occured in two $(8.70 \%)$ patients because of renal and hepatic failure. We explained low mortality rates with relative small number of our cases, providing airway safety promptly, effective diuresis and hydration, appropriate symptomatic treatment and close monitorization in the intensive care unit and early performing of dialysis and tracheostomy. In intoxications with PPD, mortality rates are found higher in the pediatric age group. These high rates can be explained by low tolerability of intoxication in childhood owing to inadequate metabolism and remove of the toxins.

In conclusion, to the best of our knowleage our study is the first in the literature including the pediatric age group only. PPD intoxication is a life-threatening situation even in low doses. Even in patients who are asymptomatic during referral patients should be carefully monitored for airway obstruction, renal, hepatic and cardiovascular complications. In the treatment, early hydration and forced diuresis is important. To prevent respiratory tract obstruction, IV steroids and when necessary IM adrenalin treatment can be applied for prophylaxis. Tracheostomy should not be avoided in necessary cases. Symptomatic treatments for organ failure and especially dialysis in case of renal failure can decrease mortality and morbidity.

\section{Acknowledgment}

The authors thank Professor Dr Behzat Ozkan for scientific contributions. 


\section{REFERENCES}

1. Bhargava P, Matthew P. Hair dye poisoning. J Assoc Physicians India 2007; 55: 871-872.

2. Sampathkumar K, Yesudas S. Hair dye poisoning and the developing world. J Emerg Trauma Shock 2009; 2: 129-131.

3. Elgamel AA, Ahmed NO. Complications and management of hair dye poisoning in Khartoum. Sudan Med Monit 2013; 8: 146-152.

4. Ahlawat G, Garg N, Kirti, Taxak S, Hooda S. Methylprednisolone in hair dye poisoning. Anaesth Pain Intens Care 2014; 18: 49-51.

5. Chrispal A, Begum A, Ramya I, Zachariah A. Hair dye poisoning-an emerging problem in the tropics: an experience from a tertiary care hospital in South India. Trop Doct 2010; 40: 100-103.

6. Kang IJ, Lee MH. Quantification of paraphenylenediamine and heavy metals in henna dye. Contact Dermatitis 2006; 55: 26-29.

7. Martin JA, Hughes TM, Stone NM. 'Black henna' tattoos: an occult source of natural rubber latex allergy? Contact Dermatitis 2005; 52: 145-146.

8. Şık G, Çıtak A. Fatal paraphenylenediamine poisoning due to black henna. Turk J Pediatr 2016; 58: 301-304.

9. Abdelraheem MB, El-Tigani MAA, Hassan EG, Ali MAM, Mohamed IA, Nazik AE. Acute renal failure owing to paraphenylene diamine hair dye poisoning in Sudanese children. Ann Trop Paediatr 2009; 29: 191-196.

10. Yagi $\mathrm{H}$, el Hind AM, Khalil SI. Acute poisoning from hair dye. East Afr Med J 1991; 68: 404-411.
11. Kallel H, Chelly H, Dammak H, et al. Clinical manifestations of systemic paraphenylene daimine intoxication. J Nephrol 2005; 18: 308-311.

12. Groholt B, Ekeberg O, Wichstrom L, Haldorsen T. Sex differences in adolescent suicides in Norway, 1990-1992. Suicide Life Threat Behav 1999; 29: 295308.

13. Foley DL, Goldston DB, Costello EJ, Angold A. Proximal psychiatric risk factors for suicidality in youth: the Great Smoky Mountains Studay. Arch Gen Psychiatry 2006; 63: 1017-1024.

14. de Groot AC. Side-effects of henna and semipermanent ' black henna ' tattoos: a full review. Contact Dermatitis 2013; 69: 1-25.

15. Chaudhary SC, Sawlani KK, Singh K. Paraphenylenediamine poisoning. Niger J Clin Pract 2013; 16: 258-259.

16. Sanchez L, Handyal H, Kannan S, et al. Hair dye poisoning: retrospective analyses of patients admitted to ICU at a rural hospital in India. Indian J Med Res 2016; 144: 134-137.

17. Kumar PAS, Talari K, Dutta TK. Super vasomol hair dye poisoning. Toxicol Int 2012; 19: 77-78.

18. Filali A, Semlali I, Ottaviano V, Furnari C, Corradini D, Soulaymani R. A retrospective study of acute systemic poisoning of paraphenylenediamine (Occidental Takawt) in Morocco. Afr J Trad CAM 2006; 3: 142-149.

19. Ram R, Swarnalatha G, Prasad N, Dakshinamurty KV. Paraphenylene diamine ingestion: an uncommon cause of acute renal failure. J Postgrad Med 2007; 53: 181-182. 